\title{
Estudo de melanoma de coróide na Universidade Federal de Uberlândia
}

\author{
Study of choroidal melanoma at the Federal University of Uberlândia
}

\author{
Enyr Saran Arcieri ${ }^{1}$ \\ Daniela Fonseca ${ }^{1}$ \\ Edimar Tiago França ${ }^{1}$ \\ Eduardo Facury Braga ${ }^{1}$ \\ Magno Antônio Ferreira ${ }^{2}$
}

\section{RESUMO}

Objetivo: Mostrar os resultados de avaliação, conduta e evolução com a população de pacientes portadores de melanoma de coróide atendidos no período de 01/01/1994 a 01/01/2000 no Ambulatório de Retina e Vítreo da Universidade Federal de Uberlândia-MG. Métodos: Análise retrospectiva de 7 prontuários de pacientes com diagnóstico de melanoma de coróide, analisando: idade, sexo, cor, sintomas e duração, métodos de diagnóstico, tratamento realizado, tamanho e tipo do tumor e evolução. Resultados: Não existiu diferença em relação ao sexo e a idade média dos pacientes foi de 58,5 anos. O tumor predominou em brancos $(66,5 \%)$ e baixa de acuidade visual foi o sintoma mais freqüente $(66,5 \%)$. O diagnóstico deveu-se principalmente à oftalmoscopia indireta $(66,5 \%)$ e todos realizaram ultrasonografia (USG). Todos pacientes foram submetidos a tratamento cirúrgico, pois apresentavam tumores grandes (maior diâmetro basal superior a $12 \mathrm{~mm}$ à USG). O tipo mais freqüente foi o de celularidade mista (50\%) e o tamanho médio dos tumores foi de $20,50 \mathrm{~mm}$ de maior diâmetro basal e $15,16 \mathrm{~mm}$ de espessura. O seguimento variou entre 8 meses e 5 anos. Dois pacientes apresentaram metástase à distância e evoluíram a óbito. Conclusões: Todos pacientes foram diagnosticados com o tumor em um estádio avançado, necessitando tratamento cirúrgico, com 2 pacientes desenvolvendo metástase à distância e evoluindo a óbito. Ressaltamos a importância da oftalmoscopia indireta em função da malignidade do tumor e da possibilidade do diagnóstico precoce, ampliando as opções de tratamento e melhorando seus resultados.

Descritores: Melanoma/diagnóstico; Neoplasias da coróide/diagnóstico
Trabalho realizado no Serviço de Oftalmologia, Departamento de Cirurgia, Faculdade de Medicina, Universidade Federal de Uberlândia - MG (UFU).

${ }^{1}$ Médicos Residentes do Serviço de Oftalmologia da UFU.

${ }^{2}$ Professor Assistente, Chefe do Setor de Retina e Vítreo, Serviço de Oftalmologia, UFU.

Endereço para correspondência: Rua. Corcovado, 155 - apto 402 - Uberlândia (MG). CEP 38411-092. E-mail: esaran@ig.com.br

\section{INTRODUÇÃO}

O melanoma maligno de coróide é o tumor primário intra-ocular mais comum em adultos, embora seja um tumor aroro $^{(1)}$, com incidência calculada em 6 casos por 1.000 .000 de habitantes por ano nos Estados Unidos ${ }^{(2-3)}$.

A apresentação é mais comum durante a 6o década de vida, sendo mais freqüente em brancos e raro em negros e asiáticos ${ }^{(3-4)}$.

Em alguns pacientes ele é assintomático, sendo um achado em exame de rotina, enquanto em outros pode causar baixa de visão, defeitos no campo visual, fotopsia ou dor.

A propedêutica principal para o diagnóstico é a oftalmoscopia indireta, e a ultra-sonografia (USG) é outro método de grande valia, pois em alguns casos somente com ela pode-se chegar ao diagnóstico, principalmente nos pacientes que apresentem opacidade de meios que impossibilitem a oftalmoscopia. 
Os tumores podem ser compostos por células fusiformes menos agressivos, células epitelióides - mais agressivos, ou células mistas - prognóstico intermediário.

Para o tratamento dos casos de melanoma de coróide, pode ser utilizado placas radioativas (Ex: iodo-125, cobalto-60), termoterapia transpupilar, enucleação, exenteração, quimioterapia ou imunoterapia, dependendo das características dos mesmos ${ }^{(4-6)}$.

Metástases à distância ou a extensão extra-escleral do tumor são as causas de morte. A sobrevida em pacientes com metástase pulmonar geralmente é menor que 1 ano, e menor que 3 meses quando há acometimento hepático ${ }^{(4)}$.

Dentre os possíveis diagnósticos diferenciais encontra$\operatorname{mos}^{(6-7)}$ : nevo de coróide, hemangioma de coróide circunscrito, tumores metastáticos para a úvea, osteoma de coróide, melanocitoma de nervo óptico, neurilemoma (schwannoma), leiomioma, hemangioma capilar da retina, hemangioma cavernoso da retina, lesões do epitélio pigmentar e tumores do epitélio não pigmentado do corpo ciliar.

Além dos tumores propriamente ditos, lesões como a degeneração senil de mácula, descolamento hemorrágico da retina, descolamento e efusão de coróide, lesões inflamatórias como esclerite posterior, granulomas corio-retinianos, lesões císticas como a retinosquise degenerativa e cistos da íris e corpo ciliar também podem simular o melanoma ${ }^{(6-7)}$.

O objetivo do presente estudo é mostrar os resultados de avaliação, conduta e evolução com a população de pacientes portadores de melanoma de coróide atendidos no Serviço de Oftalmologia - Setor de Retina e Vítreo do Hospital de Clínicas da Universidade Federal de Uberlândia - MG.

\section{MÉTODOS}

Foram analisados retrospectivamente 7 prontuários de pacientes atendidos no período de 01/01/1994 a 01/01/2000 no Serviço de Oftalmologia no Setor de Retina e Vítreo do Hospital de Clínicas da Universidade Federal de Uberlândia - MG, com diagnóstico de melanoma de coróide.

Analisamos a idade, sexo, cor, sintomas e duração destes, métodos usados para diagnóstico, tratamento realizado, tamanho e tipo do tumor e evolução dos pacientes.

O diagnóstico oftalmológico foi inferido por oftalmoscopia binocular indireta acompanhada de USG, exceto nos casos onde era impossível a realização de oftalmoscopia por opacidade de meios, sendo realizado, então, apenas a USG. As ecografias foram realizadas pelo mesmo autor (MAF), empregando-se o ecógrafo A/B scan Coopervision, com sonda de 10 MHz. A técnica de contato foi sistematicamente empregada, utilizando-se metilcelulose a $2 \%$. A varredura do globo foi realizada nos planos transversal, longitudinal e axial.

Nenhum olho foi submetido à biópsia. O diagnóstico e a medida do tamanho do tumor foram confirmados no exame anátomo-patológico da peça cirúrgica. A cirurgia foi sempre realizada sob anestesia geral, por oftalmologista experiente.
Todos os pacientes submeteram-se à avaliação clínica préoperatória, com resultado negativo quanto à pesquisa de metástases sistêmicas. Após realização da cirurgia, o acompanhamento é realizado ambulatorialmente pelos serviços de Oftalmologia e Oncologia, com avaliação sistêmica periódica incluindo exame físico, dosagem de enzimas hepáticas, realização de raio-X de tórax, USG e tomografia computadorizada de abdome, para afastar doença metastática.

\section{RESULTADOS}

Durante o período do estudo - 01/01/1994 a 01/01/2000 - 7 pacientes foram diagnosticados como portadores de melanoma de coróide. Um paciente foi eliminado deste estudo por ter optado em realizar o tratamento em outro serviço.

Os dados pertencentes aos 6 pacientes avaliados encontram-se no quadro 1.

Dos 6 pacientes avaliados, $3(50 \%)$ eram do sexo masculino e $3(50 \%)$ do sexo feminino, com idade variando entre 45 e 82 anos (média de 58,50 $\pm 15,05$ anos). Quatro $(66,5 \%)$ eram brancos e $2(33,5 \%)$ eram pardos. O período de acompanhamento desses pacientes variou entre 8 meses e 5 anos (média de 27,33 $\pm 21,67$ meses).

A sintomatologia que levou estes pacientes a procurarem atendimento médico foi baixa de visão em $4(66,5 \%)$ e associação entre dor e proptose em 1 (16,5\%). Em 1 paciente (16,5\%) o tumor foi um achado após realização de cirurgia de catarata, pois antes da cirurgia era impossível realizar oftalmoscopia e a paciente não realizou USG prévia, mas após a cirurgia de catarata foi possível realizar oftalmoscopia e USG. A duração destes sintomas variou entre 15 dias a 2 anos.

Quatro pacientes $(66,5 \%)$ foram diagnosticados através de oftalmoscopia indireta e ultra-sonografia. Em 2 (33,5\%) o diagnóstico só foi possível através da realização de USG, pois um apresentava hemorragia vítrea densa (paciente 3) e o outro tinha opacidade total de meios (paciente 1).

A refletividade interna predominante foi caracterizada ao diagnóstico pela USG como média/baixa com "ângulo Kappa" pronunciado em $5(83,5 \%)$ casos e média em $1(16,5 \%)$ caso. Escavação da coróide foi observada em $100 \%$ dos casos ao diagnóstico, com detecção de infiltração escleral pela ecografia em 1 dos casos $(16,5 \%)$.

Todos pacientes apresentavam tumores com maior diâmetro basal superior a $12 \mathrm{~mm}$ e foram submetidos a tratamento cirúrgico, sendo $5(83,5 \%)$ enucleações e $1(16,5 \%)$ exenteração no caso onde havia extensão extra-ocular do tumor.

O resultado do exame anátomo-patológico das peças (Quadro 2) evidenciou 2 (33,5\%) casos de melanoma maligno de coróide do tipo células fusiformes B, 1 (16,5\%) caso composto por células epitelióides e $3(50 \%)$ casos com células mistas. O tamanho médio dos tumores era de $20,50 \mathrm{~mm}$ de maior diâmetro basal (variação de 12 a $45 \mathrm{~mm}$ ) e 15,16 mm de espessura (variação de 7 a $25 \mathrm{~mm}$ ). 


\begin{tabular}{|c|c|c|c|c|c|c|c|c|c|}
\hline Pct & Sexo & $\begin{array}{c}\text { Id } \\
\text { anos }\end{array}$ & Cor & Sintomas & $\begin{array}{l}\text { Duração } \\
\text { Sintomas }\end{array}$ & $\begin{array}{c}\text { Método } \\
\text { Diagnóstico }\end{array}$ & Tto & $\begin{array}{l}\text { Tto } \\
\text { Comp }\end{array}$ & $\begin{array}{c}\text { Tempo } \\
\text { Seguimento }\end{array}$ \\
\hline 1 & M & 45 & B & dor + proptose & 1 ano & USG & $R t+E x$ & Qt & 2 anos * \\
\hline 2 & $\mathrm{~F}$ & 82 & B & ——- & -—- & $F O+U S G$ & En & Rt & 1 ano \\
\hline 3 & M & 55 & $\mathrm{P}$ & BAV & 15 dias & USG & En & $\mathrm{Rt}+\mathrm{Qt}$ & 4 anos * \\
\hline 4 & $\mathrm{~F}$ & 50 & $\mathrm{P}$ & BAV & 2 anos & $F O+U S G$ & En & $\mathrm{Rt}$ & 5 anos \\
\hline 5 & M & 72 & B & BAV & 2 anos & $F O+U S G$ & En & $\mathrm{Rt}$ & 1 ano \\
\hline 6 & $\mathrm{~F}$ & 47 & B & BAV & 45 dias & $F O+U S G$ & En & $\mathrm{Rt}$ & 8 meses \\
\hline
\end{tabular}

\begin{tabular}{|lcccc|}
\hline \multicolumn{2}{|c|}{ Quadro 2. Características anátomo-patológicas dos olhos de 6 pacientes portadores de melanoma maligno de coróide tratados pelo } \\
Serviço de Retina e Vítreo da Universidade Federal de Uberlândia
\end{tabular}

O paciente no 1 apresentava extensão extra-ocular do tumor, sendo submetido previamente a radioterapia externa em órbita, com dose total de 5040 cGy, e posteriormente foi submetido à exenteração. Seu tumor era composto por células fusiformes B e media $45 \mathrm{~mm}$ no maior diâmetro basal e $25 \mathrm{~mm}$ de espessura. Realizou acompanhamento no Serviço de Oncologia e 2 anos após a cirurgia foi diagnosticada metástase hepática, iniciando quimioterapia, tendo feito 2 ciclos de 3 dias cada, com intervalo de 21 dias entre os ciclos, recebendo as seguintes drogas: dacarbazina, cisplatina e hidroxiuréia, além de tamoxifeno diariamente. Após o diagnóstico da metástase hepática evoluiu a óbito em um período de 70 dias.

Os outros 5 pacientes foram submetidos à radioterapia externa em órbita após a cirurgia, recebendo uma dose total média de 6000 cGy cada.

O paciente $\mathrm{n}^{\mathrm{o}} 3$ tinha um tumor composto por células mistas, que media $16 \mathrm{~mm}$ no maior diâmetro basal e $12 \mathrm{~mm}$ de espessura, infiltrando superficialmente a esclera. Apresentou metástase pulmonar 2 anos após a enucleação e cerca de 1 ano e meio após término da radioterapia, iniciando esquema de quimioterapia com 6 ciclos de 5 dias e intervalo de 21 dias, usando dacarbazina nos 5 dias do ciclo e cisplatina no $2^{\circ}$ dia. Este paciente morreu 2 anos após o diagnóstico da metástase.

A sobrevida em nosso estudo foi de $66,5 \%$. Os pacientes no 2, 4, 5 e 6 permanecem em acompanhamento ambulatorial, com avaliação sistêmica periódica incluindo exame físico, dosagem de enzimas hepáticas, realização de raio-X de tórax, USG e tomografia computadorizada de abdome, estando estáveis e sem incidência de metástase.

\section{DISCUSSÃO}

Em nosso estudo não houve diferença significativa em relação ao sexo, reafirmando que a doença não tem afinidade especial por um ou outro $\operatorname{sexo}^{(8-9)}$. A média da idade dos pacientes analisados foi de 58,50 $\pm 15,05$ anos, coincidindo com a faixa etária de maior acometimento por este tipo de neoplasia ${ }^{(1)}$.

Houve uma maior incidência do tumor em brancos $(66,5 \%)$, não tendo encontrado nenhum paciente negro, sendo este dado coincidente com os da literatura ${ }^{(10)}$.

A queixa de perda visual é o sintoma mais freqüente nos portadores de melanoma de coróide ${ }^{(10)}$, e em nosso trabalho representou $66,5 \%$ da sintomatologia dos pacientes.

Salientamos a importância da oftalmoscopia indireta no diagnóstico, pois em nossa casuística foi possível identificar $66,5 \%$ dos casos através dela. A oftalmoscopia indireta em olhos com meios claros é o suficiente para um diagnóstico preciso de melanoma de coróide na grande maioria dos $\operatorname{casos}^{(4)}$.

O ultra-som é um exame particularmente interessante para o diagnóstico, principalmente quando há descolamento de retina extenso ou meios opacos, podendo confirmar a natureza sólida da lesão e suas dimensões exatas - o tamanho do tumor vai influir não só na escolha do tratamento, como está relacionado ao prognóstico do paciente ${ }^{(11-12)}$. Em nossa casuística todos pacientes realizaram USG, sendo esse exame de fundamental importância para o diagnóstico de 2 casos.

Os achados ultra-sonográficos em nossos pacientes coin- 
cidem com os descritos na literatura ${ }^{(13)}$, onde a maioria dos tumores caracterizaram-se por refletividade média/baixa com "ângulo Kappa" pronunciado à USG.

O melanoma maligno de coróide formado por células mistas foi o tipo de tumor mais comum em nossa amostra, sendo este achado similar aos dados do Collaborative Ocular Melanoma Study ${ }^{(14)}$.

De acordo com alguns autores ${ }^{(5)}$, a conduta nos casos de melanoma de coróide deve ser a seguinte:

1 - paciente sem qualquer sinal de doença metastática:

- tumores de até $3 \mathrm{~mm}$ de espessura - observação periódica;

- tumores de 3 a $8 \mathrm{~mm}$ de espessura e base de até $15 \mathrm{~mm}$ - braquiterapia;

- tumores com mais de $8 \mathrm{~mm}$ de espessura:

- se base de até $15 \mathrm{~mm}$ : ainda pode-se tentar braquiterapia (prognóstico reservado);

- se a base for maior que $15 \mathrm{~mm}$ : indicado enucleação.

2 - paciente com sinal de doença metastática - observação ou enucleação se o olho for doloroso.

Todos pacientes avaliados em nosso serviço apresentaram tumores grandes no momento do diagnóstico, limitando as possibilidades de tratamento, restando como solução a intervenção cirúrgica.

Alguns autores utilizam radioterapia prévia à enucleação na tentativa de reduzir o risco subseqüente de disseminação metastática ${ }^{(4)}$, o que foi realizado no paciente no 1 . A radioterapia pré-cirúrgica teria como intuito esterilizar o tumor, diminuindo o potencial reprodutivo das células tumorais que eventualmente fossem disseminadas durante o ato cirúrgico, reduzindo o número de células viáveis abaixo do necessário para reiniciar o crescimento tumoral ${ }^{(15-16)}$.

Por se tratar de um tratamento multidisciplinar, o Serviço de Oncologia dessa instituição optou por submeter os outros 5 pacientes à radioterapia externa após a realização da enucleação, na tentativa de reduzir uma provável disseminação metastática nesses pacientes, uma vez que não foi possível a prévia realização de radioterapia nesses pacientes.

Em relação ao tamanho do tumor, tem sido referida sobrevida em 20 anos de $80 \%$ para os pequenos e $40 \%$ para os grandes sem uso de radioterapia prévia ${ }^{(17)}$. Em 32 anos de seguimento, observou-se valor de sobrevida estimado em $80 \%$ para o total de pacientes enucleados ${ }^{(18)}$.

Em um estudo ${ }^{(19)}$, um grupo de pacientes portadores de melanoma de coróide, com tamanho médio de 13,3 mm no maior diâmetro basal e 10,2 mm de espessura, foi submetido à enucleação simples, com seguimento entre 6 e 155 meses. Ao final deste período, 18 pacientes estavam vivos sem doença $(62,1 \%) ; 1$ estava vivo com doença metastática em atividade (3,5\%); 6 tiveram óbito por doença metástica $(20,1 \%)$ e 4 perderam o seguimento $(13,8 \%)$.

Apesar do prognóstico ser pior nos pacientes com mais de 65 anos $^{(4)}$, os que evoluíram a óbito em nosso estudo tinham idades de 45 e 55 anos. Destes, um apresentava extensão extra-ocular do tumor ao diagnóstico e o outro tinha infiltração superficial da esclera observada no exame anátomo-patológico, confirmando o dado de que extensão extra-escleral acarreta pior prognóstico ${ }^{(4)}$.

Ambos desenvolveram metástase à distância, com acometimento hepático em um e pulmonar no outro, com sobrevida de 70 dias e 2 anos respectivamente, apesar de terem realizado tratamento quimioterápico adjuvante após o diagnóstico da metástase. $\mathrm{O}$ acompanhamento desses pacientes precisa ser longo, pois as metástases podem ocorrer até 10 anos após o diagnóstico ${ }^{(9)}$.

É importante salientar as dimensões do tumor no momento do diagnóstico, pois tendo em vista que o melanoma de coróide é um tumor de crescimento lento, levando cerca de 5 anos para atingir grandes dimensões $(>15 \mathrm{~mm})^{(20)}$, os pacientes diagnosticados em nosso serviço já apresentavam uma doença de longa data.

Acreditamos que a situação sócio-cultural desses pacientes, caracterizada pela desinformação e até mesmo à dificuldade de atendimento contribuíram para o diagnóstico da lesão em um fase avançada, limitando às opções terapêuticas e tornando frustrante a evolução de uma neoplasia ocular potencialmente tratável, pois os pacientes portadores de tumores oculares podem ter um pior prognóstico visual e sistêmico na ocorrência de atraso diagnóstico e terapêutico.

Dois fatores principais contribuem para esse $\operatorname{atraso}^{(21)}$ : a falta de informação do doente ou de seu responsável (quando se trata de criança) com relação à importância do quadro clínico, ocorrendo estreita ligação com o baixo grau de instrução, e o atraso na decisão de procurar auxílio médico decorrente da conhecida dificuldade de acesso aos serviços públicos de saúde.

Apesar da raridade, mas diante da gravidade dessa neoplasia, consideramos a relevância da oftalmoscopia indireta no diagnóstico, bem como a necessidade de um esclarecimento da população em relação à significância das alterações oculares e visuais (a exemplo do realizado na Campanha Nacional de Prevenção da Cegueira e na Campanha Olho no Olho, pois, na grande maioria dos casos, é suficiente para detectar precocemente o tumor, melhorando o prognóstico e ampliando as opções terapêuticas).

\section{ABSTRACT}

Purpose: To show the results of evaluation, management and evolution in a population of patients with choroidal melanoma that were examined between January $1^{\text {st }}, 1994$, and January $1^{\text {st }}$, 2000 at the Retina and Vitreous Service of the Federal University of Uberlândia - MG. Methods: This series was based on a retrospective chart review of 7 patients with a diagnosis of choroidal melanoma, analyzing: age, sex, race, symptoms and duration, diagnostic methods, treatment, tumor size and type, and evolution. Results: There was no difference regarding sex, and the patients' average age at diagnosis was 58.5 years. 
The tumor was more frequent in whites $(66.5 \%)$ and visual loss was the most frequent symptom $(66.5 \%)$. Indirect ophthalmoscopy was responsible for $66.5 \%$ of the diagnosis. All the patients were submitted to ultrasonography (USG) and underwent surgical treatment because they presented with large tumors (basal diameter larger than $12 \mathrm{~mm}$ ). Mixed cell melanoma was the most frequent type (50\%), and the average size of the tumors was $20.50 \mathrm{~mm}$ basal diameter and $15.16 \mathrm{~mm}$ thickness. The follow-up varied from 8 months to 5 years. Two patients had systemic metastases and died. Conclusion: All patients were diagnosed with the tumor at an advanced stage, needing surgical treatment. Two deaths were observed. Indirect ophthalmoscopy is very important in adults based on the malignancy of the tumor and the possibility of early detection, enlarging the treatment options and improving its results.

Keywords: Melanoma/diagnosis; Choroid neoplasms/diagnosis

\section{REFERÊNCIAS}

1. Schellini SA, Jorge EN, Milanezi, MFG, Marques MEA. Melanoma de coróide na criança - relato de caso e revisão da literatura. Arq Bras Oftalmol 1996;59:625-8.

2. Coleman DJ, Silverman RH, Rondeau MJ, Coleman JA, Rosberger D, Ellsworth RM et al. Ultrasonic tissue characterization of uveal melanoma and prediction of patient survival after enucleation and brachytherapy. Am J Ophthalmol 1991;112:682-8.

3. Cruickshanks KJ, Fryback DG, Nondahl DM, Robinson N, Keesey U, Dalton DS et al. Treatment choice and quality of life in patients with choroidal melanoma. Arch Ophthalmol 1999;117:461-7.

4. Kanski JJ. Tumor of the Eye. In: Kanski JJ. Clinical Ophthalmology: a systematic approach. 3rd ed. Oxford: Butterworth-Heinemann;1994. p.201-32.
5. Erwenne CM. Os métodos de tratamento e as rotinas em tumores intraoculares. Arq Bras Oftalmol 1998;61:602-7.

6. Santo RM, Bechara SJ. Tumores intra-oculares. Arq Bras Oftalmol 1998;61: 242-55.

7. Shields JA, Shields CL. Intraocular tumors: a text and atlas. Philadelphia: W.B. Saunders;1992. p.138-53.

8. Osterlind A. Trends in incidence of ocular malignant melanoma in Denmark 1943-1982. Int J Cancer 1987;40:161-4.

9. Hayton S, Lafreniere R, Jerry LM, Temple WJ, Ashley P. Ocular melanoma in Alberta: a 38 year review pointing to the importance of tumor size and tumor histology as predictors of survival. J Surg Oncol 1989;42:215-8.

10. Hill JC, Stannard C, Bowen RM. Ciliary body malignant melanoma in a black child. J Pediatr Ophthalmol Strabismus 1991;28:38-40.

11. Broadway D, Lang S, Harper J, Madanat F, Pritchard J, Tarawneh M et al. Congenital malignant melanoma of the eye. Cancer 1991;67:2642-52.

12. Betinjane AJ, Carvalho CA, Azevedo ML, Helal Jr J. Melanoma da coróide em jovem - inflamação e hipertensão intra-ocular aguda como manifestações clínicas iniciais. Arq Bras Oftalmol 1984;47:121-4.

13. Palazzi MA, Chojniak MMM. Achados ultrassonográficos em pacientes com melanoma uveal submetidos à braquiterapia. Arq Bras Oftalmol 1994;57:311-6.

14. Collaborative Ocular Melanoma Study Group. Histopathologic characteristics of uveal melanomas in eyes enucleated from the collaborative ocular melanoma study. COMS Report No. 6. Am J Ophthalmol 1998;125:745-66.

15. Char DH, Phillips TL. The potential for adjuvant radiotherapy in choroidal melanoma. Arch Ophthalmol 1982;100:257-8.

16. Powers WE, Palmer LA. Biologic basis of pre-operative radiation treatment. Radiology 1968;102:176-92.

17. Gamel JW, Mclean IW, Greenberg RA. Interval-by-interval Cox-Model analysis of 3.680 cases of intraocular melanoma shows a decline in the prognostic value of size and cell type over time after tumor excision. Cancer 1988;61: 574-9.

18. Migdal C. Effect of the method of enucleation on the prognosis of choroidal melanoma. Br J Opthalmol 1983;67:385-8

19. Chojniak MMM, Salvajoli JV, Saba LB, Palazzi MA, Freitas MAF, Mendonça $\mathrm{R}$ et al. Melanoma de coróide: estudo de sobrevida - Enucleação simples x Enucleação com radioterapia prévia. Arq Bras Oftalmol 1995;58:50-5.

20. Sahel JA, Pesavento R, Frederick AR, Albert DM. Melanoma arising de novo over a 16-month period. Arch Ophthalmol 1988;106:381-5.

21. Baddini-Caramelli C, Rotenberg M, Avakian A, Silva ALB, Damico FM, Takei LM et al. Tumores oculares: fatores de atraso no atendimento oftalmológico. Arq Bras Oftalmol 1997;60:604-7.

\title{
ABO ELETRÔNICO
}

\section{A versão eletrônica dos Arquivos Brasileiros de}

\section{Oftalmologia com textos completos está disponível em:}

\author{
- $\mathrm{ABO}$ - Arquivos Brasileiros de Oftalmologia \\ http://www.abonet.com.br
}

- SciELO - Scientific Electronic Library Online http://www.scielo.org

- Free Medical Journals - http://www.freemedicaljournals.com 